Astrophysical Applications of Stellar Pulsation

ASP Conference Series, Vol. 83, 1995

R. S. Stobie and P. A. Whitelock, eds.

\title{
Cepheid Radii and Distances
}

\author{
C. D. Laney and R. S. Stobie
}

South African Astronomical Observatory, PO Box 9, Observatory, 7935, Western Cape, South Africa

Laney \& Stobie (1995) (LS95) have determined Baade-Wesselink radii for 49 galactic Cepheids using BVRI and JHK photometry. Four of the possible magnitude-colour combinations were studied in detail - namely, (V,B-V), (V,V$\left.\mathrm{I}_{\mathrm{C}}\right),(\mathrm{K}, \mathrm{V}-\mathrm{K})$ and $(\mathrm{K}, \mathrm{J}-\mathrm{K})$. In that paper and in the present note we have used the maximum likelihood method of Balona (1977) as recently modified to allow for the nonlinear effects of the non-negligible radius excursions of Cepheids.

One major advantage of infrared photometry is that the light variation at $\mathrm{K}$ is dominated by the change in surface area, unlike the light variation at $\mathrm{V}$ which is dominated by temperature changes. Another is that (as shown both empirically and by model atmospheres) one may use a J-K or $\mathrm{V}-\mathrm{K}$ colour index to calculate the surface brightness at $\mathrm{K}$ without having to worry very much about the effects of varying surface gravity or Doppler broadening velocity, while the same is definitely not true when optical colour indices are used to calculate the surface brightness at $\mathrm{V}$.

From radius displacement curves it is clear that the systematic and random errors are least in the case of the infrared solutions, $(\mathrm{K}, \mathrm{V}-\mathrm{K})$ and $(\mathrm{K}, \mathrm{J}-\mathrm{K})$. $(\mathrm{V}, \mathrm{B}-\mathrm{V})$ and $\left(\mathrm{V}, \mathrm{V}-\mathrm{I}_{\mathrm{C}}\right)$ solutions have large systematic and random errors. Furthermore, "optical" radius determinations appear quite sensitive to whether the phase of the ascending branch is excluded from the solution. "Infrared" solutions show no such effect.

The most accurate period-radius relation was derived by averaging the $(\mathrm{K}, \mathrm{V}-\mathrm{K})$ and $(\mathrm{K}, \mathrm{J}-\mathrm{K})$ radius solutions. $(\mathrm{V}, \mathrm{B}-\mathrm{V})$ and $(\mathrm{V}, \mathrm{V}-\mathrm{I})$ solutions gave systematically incorrect radius determinations because of the effects of surface gravity and microturbulence variations throughout the pulsation cycle. (V,V$R_{J}$ ) radius solutions (calculated for about half of our sample) showed systematic errors essentially identical to those seen in $\left(\mathrm{V}, \mathrm{V}-\mathrm{I}_{\mathrm{C}}\right)$ solutions.

Given the availability of radial velocities contemporaneous with our JHK data for LMC and SMC Cepheids, we have calculated "infrared" radii for as many Cepheids as possible, despite the limited quality and limited precision of the data compared to our galactic sample. Contemporaneous radial velocities were available in the literature. Radius solutions were considered successful only if the surface brightness coefficients were within 0.6 of the expected value at a given period. No solutions for Cepheids with periods longer than 100 days were successful. For 5 LMC and 4 SMC Cepheids (HV 12815, 879, 2338, 2827, 5497, $837,824,11157$, and 829), radii could be determined, and the adopted radii are plotted in Fig. 1, together with our "adopted" galactic Cepheid radii. The LMC and SMC Cepheid radii show no systematic difference from the galactic Cepheid 


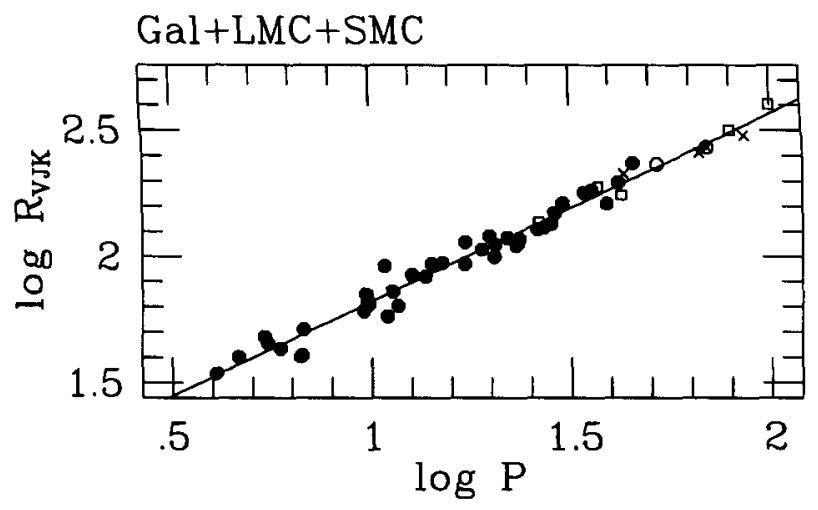

Figure 1. $\log R-\log P$ relation for Cepheids in the Galaxy (circles), LMC (squares) and SMC (crosses).

radii and a combined Galaxy/LMC/SMC period-radius relation

$$
\begin{aligned}
\log R= & 1.821+0.747(\log P-1)+0.005(L M C)-0.009(S M C) & \sigma=0.043 \\
& \pm 0.008 \pm 0.023 \quad \pm 0.024 \quad \pm 0.027 &
\end{aligned}
$$

is the same as within the errors as the (galactic) relation given in LS95, and suggests that (again within the errors) the galactic and MC bolometric PL relations are not significantly different.

Absolute magnitudes for 37 Cepheids (V) and 40 Cepheids (JHK) have been calculated using the tables for surface brightness given by Hindsley \& Bell $(1989,1990)$. Combining these with the dereddened mean magnitudes for 45 LMC and 47 SMC Cepheids with VJHK photometry enables period-luminosity relations in each colour to be derived with effective "distance moduli" for the LMC and SMC. Corrected for the effects of mean difference in temperature at a given period and for the differences in blanketing between galactic and Cloud Cepheids (as in Laney \& Stobie 1994), we find that the corrected VJHK moduli are in agreement, with means of $18.58 \pm 0.04$ for the LMC and $19.00 \pm 0.04$ for the SMC. These mean values are in remarkably good agreement with the moduli of 18.53 and 18.94 we found using the galactic Cepheids in clusters and associations together with the same sample of Cloud Cepheids (Pleiades modulus 5.57).

\section{References}

Balona, L.A. 1977, MNRAS, 178, 231

Hindsley, R.B., \& Bell, R.A. 1989, ApJ, 341, 1004

Hindsley, R.B., \& Bell, R.A. 1990, ApJ, 348, 673

Laney, C.D., \& Stobie, R.S. 1994, MNRAS, 266, 441

Laney, C.D., \& Stobie, R.S. 1995, MNRAS, in press 